\title{
Investigation of a special neutralizing epitope of HEV E2s
}

\section{Dear Editor}

After 14-years of development, the first prophylactic vaccine against the Hepatitis E virus (HEV) has been marketed since 2012 (Wu et al., 2012). However, the neutralizing epitopes of HEV are not completely defined. E2s, a protruding homodimer domain of HEV capsid protein, is responsible for interacting with host cells to initiate infection ( $\mathrm{Li}$ et al., 2009; $\mathrm{Li}$ et al., 2005). It was shown that two monoclonal antibodies $(\mathrm{mAb}), 8 \mathrm{C} 11$ and $8 \mathrm{H} 3$, could neutralize the infectivity of HEV in rhesus, and the two mAbs bind to different neutralizing conformational epitopes on E2s (Zhang et al., 2005). The epitope for $8 \mathrm{C} 11$ was reported based on the crystal structure of HEV protruding domain E2s in complex with 8C11 Fab (Tang et al., 2011). In a HEV antibody panel consisting of 30 mAbs, including $8 \mathrm{C} 11$, none of them can cross block the binding of $8 \mathrm{H} 3$ to $\mathrm{E} 2 \mathrm{~s}$. These findings suggest that $8 \mathrm{H} 3$ recognizes a special epitope which is different from the epitope for $8 \mathrm{C} 11$ and other mAbs. X-ray crystallography is not a viable option to identify the $8 \mathrm{H} 3$ epitope because $8 \mathrm{H} 3$ binds to the E2s with a low affinity (Zhang et al., 2005).

Conformational epitopes, which often escape identification by linear peptide screening, can be identified and characterized from studies with mimotopes (Cardoso et al., 2009). Most mimotopes obtained from phage displayed peptide libraries can be employed to facilitate the identification of novel peptide sequences that mimic binding sites for mAbs (Mayrose et al., 2007). We panned three phagedisplayed peptide libraries (ph.D.-C7C and ph.D.-12 displaying peptide on the plll protein, and lib $\mathrm{C} 10 \mathrm{C}$ displaying peptide on the pVIII protein) to select $8 \mathrm{H} 3$ mimotopes (Table S1). After three rounds of panning, phage clones were tested for binding specificity to $8 \mathrm{H} 3$. Mimotopes which reacted to $8 \mathrm{H} 3$ without cross-reacting with three HEV related antibodies $(8 \mathrm{C} 11,12 \mathrm{G} 8$, and $8 \mathrm{G} 12)$ and two HEV non-related antibodies (13D4 against AIV and 42B6 against $\mathrm{HBV}$ ) were considered positive. Finally, 21 mimotopes to $8 \mathrm{H} 3$ were obtained for further analysis (Table S2).

The 21 mimotopes to the $8 \mathrm{H} 3 \mathrm{mAb}$ were processed individually by three efficacious prediction programs, Pep3D-Search program, Pepsurf and EpiSearch for $8 \mathrm{H} 3$ epitope mapping (Huang et al., 2008; Mayrose et al., 2007; Negi and
Braun, 2009). The E2s structure of the HEV (PDB: 3GGQ) was used as a template for epitope prediction ( $\mathrm{Li}$ et al., 2009). The mimotope sequences listed in Table S2 were used to deduce the best cluster by default parameters. The prediction results from the three programs were shown in Table S3. Overlapping regions of the predicted clusters from the three programs, composed of $\mathrm{Gln}^{482}-\mathrm{Thr}^{484}$, $\mathrm{Ser}^{487}$. Gly $^{490}$, Ser $^{527}-$ Pro $^{540}$, Tyr $^{559}-$ Asn $^{560}$, Asn $^{562}-$ GIn $^{568}, \mathrm{Asn}^{573}$ and $\mathrm{Ser}^{582}-\mathrm{Thr}^{586}$, were considered parts of the $8 \mathrm{H} 3$ epitope. These overlapping regions (shown in rose red in Fig. $1 \mathrm{~A}$ ) are located on the groove of E2s, and they are independent from the epitope of $8 \mathrm{C} 11$.

The prediction results based on the mimotope sequences provide general information on the binding region on the antigen, but it does not provide the details on antigen-antibody contacts, for example, the amino acids involving in hydrogen-bonding contacts and the binding sites on the antibody. The rigid-body protein-protein docking program ZDOCK was then used to map the antigen-antibody contact sites. Fast Fourier Transform (FFT) algorithm was applied to perform a global docking to search for potential binding positions of two component proteins (Pierce et al., 2014). Since validity of the ZDOCK analysis is affected by the accuracy of the search algorithm as well as the proteinprotein complex to be predicted, some of the top-scoring predictions resulted from the soft scoring function of the program could be false positives (Wiehe et al., 2008). Combining the results from epitope prediction softwares based on mimotope and ZDOCK may lead to a more reliable result. The overlapping regions $\left(\mathrm{Gln}^{482}-\mathrm{Thr}^{484}\right.$, Ser ${ }^{487}$ $\mathrm{Gly}^{490}, \mathrm{Ser}^{527}$-Pro $^{540}, \mathrm{Tyr}^{559}-\mathrm{Asn}^{560}, \mathrm{Asn}^{562}{ }_{-\mathrm{Gln}^{568}}{ }^{56 \mathrm{As}^{573}}$ and $\mathrm{Ser}^{582}-\mathrm{Thr}^{586}$ ) predicted from the three programs were further investigated by ZDOCK. The 3-dimensional model of mAb $8 \mathrm{H} 3$ was generated by a homology modeling protocol. Given the facts that the epitope of antibody $8 \mathrm{H} 3$ is different from that of $8 \mathrm{C} 11$, and the binding of $8 \mathrm{H} 3$ to $\mathrm{E} 2 \mathrm{~s}$ can be enhanced by $8 C 11$ (Zhang et al., 2005), the structure of 8C11 Fab-E2s complex (PDB: 3RKD) was used as the antigen for the ZDOCK program to search for the best combination model. As a result, the surface on antigen E2s for binding to antibody $8 \mathrm{H} 3$ were shown in red (Fig. 1B). The regions of E2s binding to $8 \mathrm{H} 3$ were further analyzed and shown in dark shade (Fig. 1C). In addition to the same three 


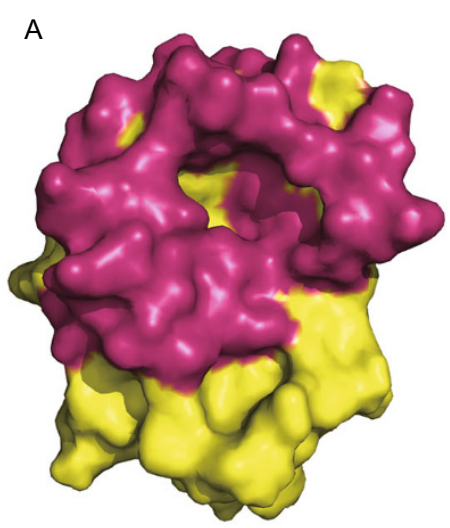

C

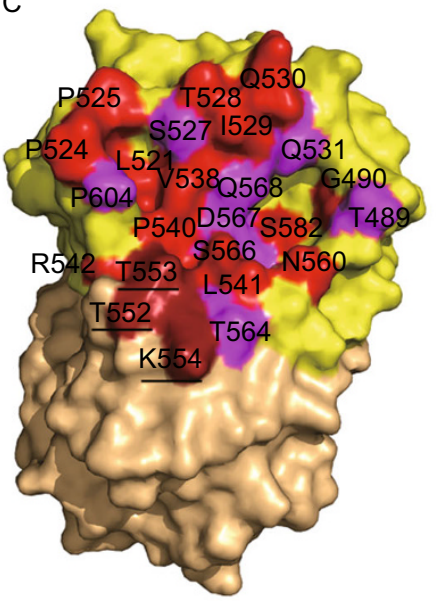

B

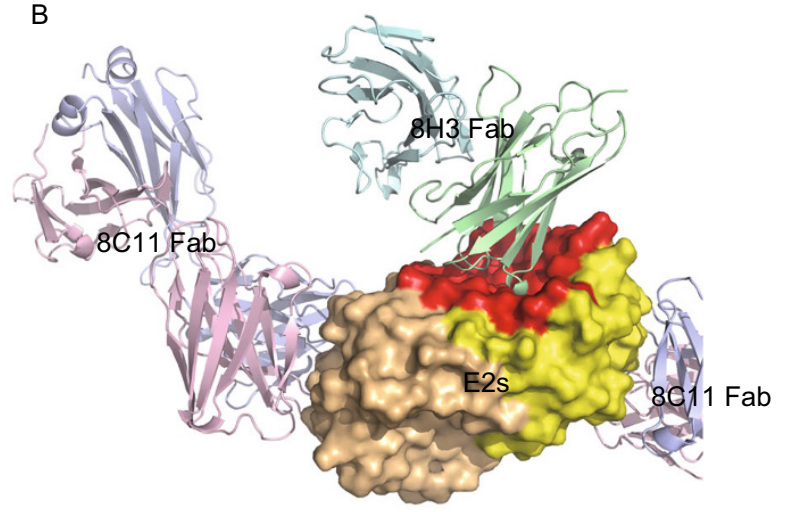

E

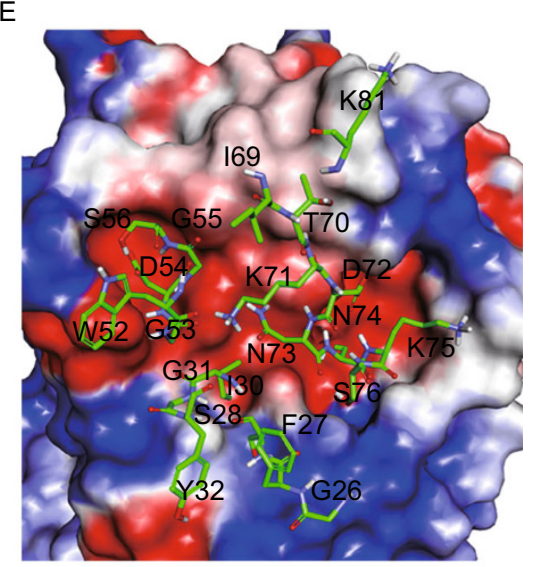

Figure 1. The predicted binding-region of $8 \mathrm{H} 3-\mathrm{E} 2 \mathrm{~s}$. (A) The sites in rose red are the overlapping epitope regions from the prediction results of three programs, Pepsurf, Pep-3D-Search, and Epi-search. (B) The predicted binding-region (in red) of 8H3-E2s (E2s, PDB: 3KRD) by ZDOCK. (C) Surface representations of E2s highlighted the interacting epitope residues in dark shade. The epitope residues shown in purple correspond to their interactions to $8 \mathrm{H} 3$ by hydrogen bonds. The epitope residues shown in brown are located on the second subunit (in orange) of E2s. (D) The major clusters of $8 \mathrm{H} 3$ epitope on E2s are shown in ball and stick model and colored in deep purple. The epitope residues labeled with underline are located on the second subunit of E2s. The 8H3 Fab is shown as surface representation, $\mathrm{H}$-chain is shown in light blue, and L-chain is green. (E) Depicts the electrostatic potential surface of the epitope on the E2s (red, negative; blue, positive; and gray, neutral) with the key residues for interaction from $8 \mathrm{H} 3 \mathrm{Fab}$ represented as sticks. The figure was prepared using the program PyMOL (Delano, 2002).

regions $\left(\mathrm{Ser}^{527}-\mathrm{Thr}^{535}, \mathrm{Tyr}^{559}-\mathrm{Asn}^{560}\right.$, and $\mathrm{Tyr}^{584}-\mathrm{Thr}^{586}$ ) predicted by the three programs based on mimotopes, there are four additional binding regions (Leu ${ }^{521}, \mathrm{Arg}^{524}-\mathrm{Pro}^{525}$, $\mathrm{Leu}^{570}$, $\mathrm{Ala}^{602}-\mathrm{PrO}^{604}$ ) on the first subunit and one region $\left(\mathrm{Thr}^{552}\right.$-Lys $^{554}$ ) on the second subunit from the prediction by ZDOCK (Fig. 1C and 1D). All the predicted interaction regions are located on the groove or near-groove of the E2s dimer structure.

In the predicted model from ZDOCK, the specificity of $8 \mathrm{H} 3$ Fab to E2s is dictated by seven major interactions at $\mathrm{Thr}^{489}$. $\mathrm{Gly}^{490}, \mathrm{Arg}^{524}-\mathrm{GIn}^{531}, \mathrm{Pro}^{540}, \mathrm{Asn}^{560}, \mathrm{Thr}^{564}-\mathrm{GIn}^{568}, \mathrm{~S}^{582}$, and $\mathrm{Ala}^{602}-\mathrm{Pro}^{604}$ of E2s (Fig. 1C and 1D). There are six hydrogen-bonding contacts from $\mathrm{Thr}^{489}$, $\mathrm{Ser}^{527}, \mathrm{Gln}^{531}$, $\mathrm{Thr}^{564}, \mathrm{GIn}^{568}$ and $\mathrm{Pro}^{604}$ of E2s to $8 \mathrm{H} 3$ Fab (Fig. 1C and
1D). Three hydrophobic loop regions of $8 \mathrm{H} 3$ heavy chain ${ }^{\mathrm{H} 26} \mathrm{GFS}^{\mathrm{H} 28}$, ${ }^{\mathrm{H} 52} \mathrm{WGDGS}^{\mathrm{H} 56}$, and ${ }^{\mathrm{H} 71} \mathrm{KDN}^{\mathrm{H73}}$ are in close contact with E2s (Fig. 1E).

To verify the prediction results functionally, all predicted sites on E2s with solvent accessibility were individually mutated to Ala and the mutants were expressed in E. coli. The wild type E2s and its mutants were subjected to nonreducing SDS-PAGE and Western blotting against $8 \mathrm{H} 3$. All samples except Thr564Ala and Ser582Ala were resolved mainly as dimers (Fig. 2A). In previous studies, we have demonstrated that the dimerization of E2s is essential for HEV-host interactions and antibody neutralization, and $\mathrm{Thr}^{564}$ and $\mathrm{Ser}^{582}$ can stabilize the dimer (Li et al., 2009; Li et al., 2005). Therefore, the Thr564Ala and Ser582Ala 
A
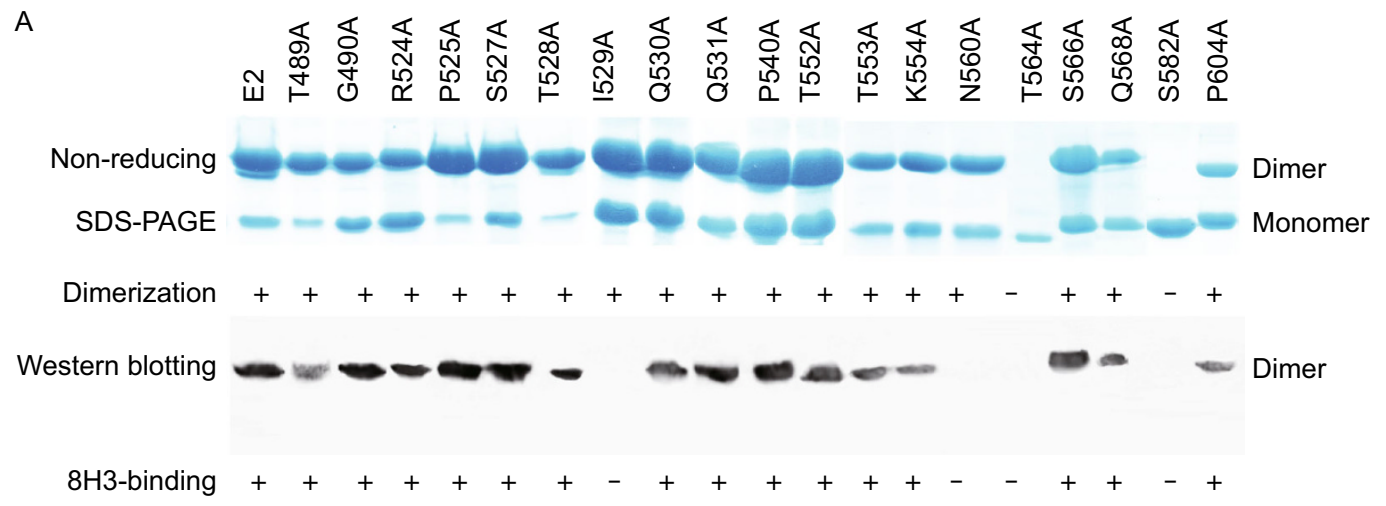

B

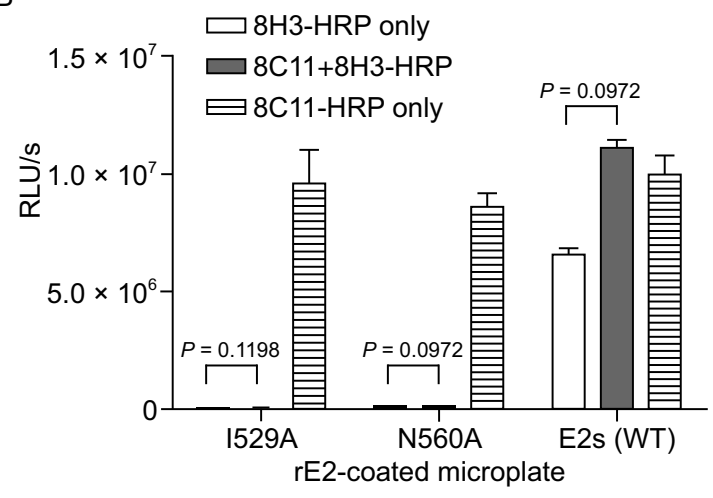

C

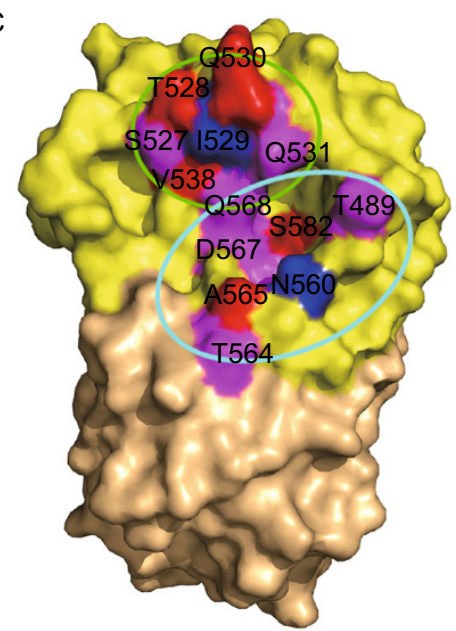

Figure 2. Functional validation of the predicted epitope of $8 \mathrm{H} 3$. (A) Mutational studies on the predicted binding-region of $8 \mathrm{H} 3$. All residues from the predicted binding-region were mutated to Ala. The wild type E2s and its mutants were subjected to non-reducing SDS-PAGE and Western blotting with $8 \mathrm{H} 3$. [+] denotes dimerization or reactivity with $8 \mathrm{H} 3,[-]$ denotes loss of dimerization or reactivity with $8 \mathrm{H} 3$. (B) Effects of $8 \mathrm{C} 11$ on the binding of $8 \mathrm{H} 3$ with $\mathrm{E} 2 \mathrm{~s}$ mutants. (C) Residues around the two key amino acid residues in dark blue $1 \mathrm{e}^{529}$ and $\mathrm{Asn}{ }^{560}$ in $6 \AA$ are circled in light green and light blue, respectively. The sites in red and purple are the predicted epitope of $8 \mathrm{H} 3$ and the residues with interactions to $8 \mathrm{H} 3$ by hydrogen bonds are shown in purple.

mutants lose the binding ability to $8 \mathrm{H} 3$ due to the collapse of dimeric form of E2s. The Western blotting results showed that the wild type dimeric E2s and 15 mutants, including the ones with mutated sites $\left(\mathrm{Thr}^{489}, \mathrm{Ser}^{527}, \mathrm{Gln}^{531}, \mathrm{Gln}^{568}\right.$ and $\mathrm{Pro}^{604}$ ) responsible for binding to $8 \mathrm{H} 3$ by hydrogen-bond as the prediction results of $Z D O C K$, were reactive with $8 \mathrm{H} 3$ (Fig. 2A). Since lle529Ala and Asn560Ala mutants in dimeric form abolished the $8 \mathrm{H} 3$ reactivity, Il ${ }^{529}$ and $\mathrm{Asn}^{560}$ contribute to the binding of $8 \mathrm{H} 3$ with E2s (Fig. 2A).

We have shown previously that mAb $8 \mathrm{C} 11$ enhances the binding of $8 \mathrm{H} 3$ to E2s (Zhang et al., 2005). If $8 \mathrm{C} 11$ can't enhance the binding of $8 \mathrm{H} 3$ to the E2s mutants, the E2s mutants can be assumed of losing the binding capacity to $8 \mathrm{H} 3$ and the epitope of $8 \mathrm{H} 3$ on $\mathrm{E} 2 \mathrm{~s}$ was destroyed by the site mutations. In order to investigate the enhancement of $8 \mathrm{C} 11$ on the binding of $8 \mathrm{H} 3$ to the lle529Ala or Asn560Ala mutants, CLIA (chemiluminescent immunoassay) was carried out using microplates coated with purified E2s mutants and pre- incubated with buffer containing $8 \mathrm{C} 11$, then reacted with HRP labeled $8 \mathrm{H} 3$. Binding of the labeled antibodies was measured in RLU (relative light unit). The results showed that the Ile529Ala and Asn560Ala mutants retained the binding activity to $8 \mathrm{C} 11$, but they can't bind to $8 \mathrm{H} 3$ with the presence of $8 \mathrm{C} 11$ (Fig. 2B), suggesting that the epitope of $8 \mathrm{C} 11$ was retained, but the epitope of $8 \mathrm{H} 3$ was damaged in mutants lle529Ala and Asn560Ala. From the structure of E2s (PDB: 3GGQ or 3RKD), we found that the amino acid residues around $l 1 e^{529}$ in $6 \AA$ included $\mathrm{Gln}^{530}, \mathrm{Thr}^{528}, \mathrm{Gln}^{531}, \mathrm{Ser}^{527}, \mathrm{Val}^{538}$ and $\mathrm{Gln}^{568}$, and residues around $\mathrm{Asn}^{560}$ in 6 A included $\mathrm{Thr}^{489}{ }^{4{ }^{2}} \mathrm{Ser}^{582}, \mathrm{Asp}^{567}$, $\mathrm{Ala}^{565}$ and $\mathrm{Thr}^{564}$ (Fig. 2C). The mutations of Asn560Ala and Ile529Ala may have resulted in conformational changes of the $8 \mathrm{H} 3$ epitope, especially those amino acid residues located around $\mathrm{Asn}^{560}$ and $1 \mathrm{e}^{529}$ in $6 \AA$. These results collectively indicated that $l l \mathrm{e}^{529}$ and $\mathrm{Asn}^{560}$ were the key sites to maintain the epitope of $8 \mathrm{H} 3$ and confirmed the prediction results that $8 \mathrm{H} 3$ targets the groove region of E2s. 
In summary, we predicted the epitope of $8 \mathrm{H} 3$ on E2s by epitope prediction softwares based on the combined approaches of mimotopes and ZDOCK. The antibody $8 \mathrm{H} 3$ recognizes the epitope composed of $\mathrm{Thr}^{489}$-Gly ${ }^{490}$, $\mathrm{Arg}^{524}$ Pro $^{525}$, Ser $^{527}-\mathrm{Gln}^{531}$, Pro ${ }^{540}$, Asn ${ }^{560}, \mathrm{Thr}^{564}-\mathrm{Gln}^{568}, \mathrm{Ser}^{582}$, and $\mathrm{Ala}^{602}$-Pro ${ }^{604}$ on the groove regions of the HEV E2s. Mutation analysis demonstrated that $\mathrm{Il}^{529}$ and $\mathrm{Asn}^{560}$ on E2s were the most crucial residues for its interaction with $8 \mathrm{H} 3$, and they were the key sites to maintain the epitope of $8 \mathrm{H} 3$. Our data presented here reveals for the first time a special nondominant neutralizing epitope located on the groove region of $\mathrm{E} 2 \mathrm{~s}$. Because $8 \mathrm{H} 3$ binds to $\mathrm{E} 2 \mathrm{~s}$ region of HEV which has been proposed to mediate the first contact with host cells to initiate viral infection ( $\mathrm{He}$ et al., 2008; $\mathrm{Li}$ et al., 2009; Tang et al., 2011), our results may have broad implications in understanding the protection mechanism of HEV vaccines and drugs designed against the HEV virus. Additionally, this is the first report of epitope mapping by combining the prediction results from mimotopes and ZDOCK. This approach may be particularly effective in investigations of special epitopes.

\section{FOOTNOTES}

This project was supported by National Major Scientific and Technological Special Project for "Significant New Drugs Development" (2013ZX09101017), Policy Guidance Project for "Institute Reconstruction Fund" (2011FU125Z04) and Xiamen City Municipal platform fund on Viral Biotherapeutics (3502Z20131001).

Min You, Lu Xin, Yi Yang, Xiao Zhang, Yingwei Chen, Hai Yu, Shaowei Li, Jun Zhang, Zhiqiang An, Wenxin Luo, and Ningshao Xia declare that they have no conflict of interest. This article does not contain any studies with human or animal subjects performed by any of the authors.

Min You ${ }^{1}$, Lu Xin ${ }^{1}$, Yi Yang ${ }^{1}$, Xiao Zhang $^{1}$, Yingwei Chen ${ }^{3}$, Hai $\mathrm{Yu}^{1}$, Shaowei $\mathrm{Li}^{1}$, Jun Zhang ${ }^{1}$, Zhiqiang $\mathrm{An}{ }^{1,2}$, Wenxin Luo $^{1 \bowtie}$, Ningshao Xia ${ }^{1}$

${ }^{1}$ National institute of diagnostics and vaccine development in infectious diseases, Xiamen University, Xiamen 361105, China

2 Texes Therapeutics Institute, The Brown Foundation of Molecular Medicine, University of Texas Health Science Center at Houston, Houston TX77030, USA

${ }^{3}$ Potomac Affinity Proteins, 9601 Medical Center Drive, Suite \#330, Rockville, MD 20850, USA

$\square$ Correspondence: wxluo@xmu.edu.cn (W. Luo)

\section{OPEN ACCESS}

This article is distributed under the terms of the Creative Commons Attribution License which permits any use, distribution, and reproduction in any medium, provided the original author(s) and the source are credited.

\section{REFERENCES}

Cardoso R, Homsi-Brandeburgo MI, Rodrigues VM, Santos WB, Souza GL, Prudencio CR, Siquieroli AC, Goulart LR (2009) Peptide mimicking antigenic and immunogenic epitope of neuwiedase from Bothrops neuwiedi snake venom. Toxicon 53:254261

Delano W (2002) The PyMOL molecular graphics system. Delano Scientific, San Carlos

He SZ, Miao J, Zheng ZZ, Wu T, Xie MH, Tang M, Zhang J, Ng MH, Xia NS (2008) Putative receptor-binding sites of hepatitis $E$ virus. J Gen Virol 89:245-249

Huang YX, Bao YL, Guo SY, Wang Y, Zhou CG, Li YX (2008) Pep3D-Search: a method for B-cell epitope prediction based on mimotope analysis. BMC Bioinform 9:538

Li SW, Zhang J, He ZQ, Gu Y, Liu RS, Lin J, Chen YX, Ng MH, Xia NS (2005) Mutational analysis of essential interactions involved in the assembly of hepatitis $E$ virus capsid. J Biol Chem 280:3400-3406

Li SW, Tang XH, Seetharaman J, Yang CY, Gu Y, Zhang J, Du HL, Shih JW, Hew CL, Sivaraman J, Xia NS (2009) Dimerization of hepatitis $E$ virus capsid protein E2s domain is essential for virushost interaction. PLoS Pathog 5:e1000537

Mayrose I, Shlomi T, Rubinstein ND, Gershoni JM, Ruppin E, Sharan R, Pupko T (2007) Epitope mapping using combinatorial phagedisplay libraries: a graph-based algorithm. Nucleic Acids Res 35:69-78

Negi SS, Braun W (2009) Automated detection of conformational epitopes using phage display peptide sequences. Bioinform Biol Insights 3:71-81

Pierce BG, Wiehe K, Hwang H, Kim BH, Vreven T, Weng Z (2014) ZDOCK server: interactive docking prediction of protein-protein complexes and symmetric multimers. Bioinformatics 12:17711773

Tang XH, Yang CY, Gu Y, Song CL, Zhang X, Wang YB, Zhang J, Hew CL, Li SW, Xia NS, Sivaraman J (2011) Structural basis for the neutralization and genotype specificity of hepatitis $E$ virus. Proc Natl Acad Sci USA 108:10266-10271

Wiehe K, Peterson MW, Pierce B, Mintseris J, Weng Z (2008) Protein-protein docking: overview and performance analysis. Methods Mol Biol 413:283-314

Wu T, Li SW, Zhang J, Ng MH, Xia NS, Zhao QJ (2012) Hepatitis E vaccine development: a 14 year odyssey. Hum Vaccines Immunother 8:823-827

Zhang J, Gu Y, Ge SX, Li SW, He ZQ, Huang GY, Zhuang H, Ng MH, Xia NS (2005) Analysis of hepatitis E virus neutralization sites using monoclonal antibodies directed against a virus capsid protein. Vaccine 23:2881-2892

Electronic supplementary material The online version of this article (doi:10.1007/s13238-014-0115-3) contains supplementary material, which is available to authorized users. 